\title{
Pion and Sigma Polarizabilities and Radiative Transitions
}

\author{
Murray A. Moinester ${ }^{1}$ \\ ${ }^{1}$ School of Physics and Astronomy \\ Raymond and Beverly Sackler Faculty of Exact Sciences, \\ Tel Aviv University, 69978 Ramat Aviv, Israel, \\ e-mail: murray tauphy.tau.ac.il \\ Bulletin Board: hep-ph xxx.lanl.gov/9409463 \\ Tel Aviv U. preprint TAUP 2204-94 \\ Contribution to Proceedings of Workshop on Chiral Dynamics, \\ Massachusetts Institute of Technology, July 1994, \\ Eds. A. Bernstein, B. Holstein.
}

\begin{abstract}
Fermilab E781 plans measurements of $\gamma$-Sigma and $\gamma$-pion interactions using a $600 \mathrm{GeV}$ beam of Sigmas and pions, and a virtual photon target. Pion polarizabilities and radiative transitions will be measured in this experiment. The former can test a precise prediction of chiral symmetry; the latter for $a_{1}(1260) \rightarrow \pi \gamma$ is important for understanding the polarizability. The experiment also measures polarizabilities and radiative transitions for Sigma hyperons. The polarizabilities can test predictions of baryon chiral perturbation theory. The radiative transitions to the $\Sigma^{*}(1385)$ provide a measure of the magnetic moment of the s-quark. Previous experimental and theoretical results for $\gamma \pi$ and $\gamma \Sigma$ interactions are given. The E781 experiment is described.
\end{abstract}

\section{Introduction}

Pion and $\Sigma(1189)$ polarizabilities and associated radiative transitions will be measured in the Fermilab E781 SELEX experiment [1,2]. Hadron electric $(\alpha)$ and magnetic $(\beta)$ intrinsic polarizabilities $[2-6]$ characterize the induced transient dipole moments of hadrons subjected to external oscillating electric $\boldsymbol{E}$ and magnetic $\boldsymbol{H}$ fields. The dipole moments are given by $\boldsymbol{d}=\alpha \boldsymbol{E}$ and by $\boldsymbol{\mu}=\beta \boldsymbol{H}$. The polarizabilities can be obtained from precise measurements of the gamma-hadron Compton scattering differential cross 
section. They probe the rigidity of the internal structure of baryons and mesons, since they are induced by the rearrangement of the hadron constituents via action of the photon electromagnetic fields during scattering. For the light charged pion, chiral symmetry leads to a precise prediction for the polarizabilities $[7,8]$. The experimental polarizabilities therefore subject the chiral perturbation techniques of QCD to new and serious tests. The availability of high energy $\Sigma$ beams raises the possibility of investigating their Compton scattering polarizabilities and radiative transitions. The $\Sigma$ polarizabilities can test predictions of baryon chiral perturbation theory [9]. The radiative transitions to the $\Sigma^{*}(1385)$ can provide a valuable measure of the magnetic moment of the s-quark $[10,11]$.

\section{Sigma Polarizabilities and Radiative Transitions}

Electromagnetic interactions of $\Sigma$ 's can be studied $[2,11]$ in FNAL experiment E781 with high energy $\Sigma$ beams via the Primakoff interaction of an incident $\Sigma$ with a virtual photon in the Coulomb field of a target nucleus of atomic number Z. The $\gamma \Sigma$ Compton scattering and associated polarizabilities are studied $[2,11]$ by detecting the final state $\gamma$-ray and $\Sigma$ in coincidence. The final state Sigma or other charged particles will be measured $[1,2]$ in magnetic spectrometers, while final state $\gamma$-rays will be measured with lead glass electromagnetic calorimeters. The laboratory decay length $(L=\gamma \beta c \tau)$ for very high energy $\Sigma$ 's exceeds ten meters, so that magnetic detection is possible. Transition radiation detectors upstream of the target and ring Cerenkov detectors downstream will provide particle identification [1]. The decay signature for $\Sigma^{*}(1385) 3 / 2^{+}$production is clean as it decays to $\Lambda \pi$, and the $\Lambda$ to $\pi^{-}$p. Such radiative transition studies were first suggested by Lipkin [10] and considered in more detail afterwards [2,11 - 15]. The $\Sigma^{0} \rightarrow \Lambda \gamma$ radiative transition (lifetime) was measured in this way [15] using a $\Lambda$ beam, and this to date is the only precision hyperon radiative transition measured. Because radiative transitions involve the well understood electromagnetic process, they provide precision tests of the quark wave functions characterizing the configurations of the low lying excited states of hadrons.

The $\Sigma^{+}$(uus) and $\Sigma^{-}$(dds) baryons are of interest because electromagnetic observables are particularly sensitive to their underlying quark structure [11]. The $\Sigma^{+}$differs from the proton only by replacing the $d$ quark by a strange quark which also has charge $-1 / 3$. Thus, any difference between the electromagnetic properties of the $\Sigma^{+}$and proton can only arise from flavor $\mathrm{SU}(3)$ symmetry breaking. The two $\Sigma$ states are isospin mirrors of one another, symmetrically placed in the same flavor $\mathrm{SU}(3)$ octet and have very similar strong interactions. However, their electromagnetic interactions are completely different because the $\Sigma^{+}$, like the nucleon and $\Xi^{o}$, have valence quarks of two flavors having the opposite sign of electric 
charge. External electromagnetic fields therefore exert forces in opposite directions on the two flavors and rotate spins in opposite directions, thereby producing internal excitation. The $\Sigma^{-}$on the other hand, and also the $\Xi^{-}$, have three valence quarks all with charge $-1 / 3$, and the principle effect of an external electromagnetic field is to exert forces in the same direction on all three valence quarks and rotate their spins in the same direction. This produces no internal excitation. We discuss below experimental implications of this effect, first noted [10] by a selection rule involving the U-spin $\mathrm{SU}(2)$ subgroup of $\mathrm{SU}(3)$. U-spin is conserved to all orders in any combination of electromagnetic interactions and strong interactions invariant under $\mathrm{SU}(3)$. The resulting $\mathrm{SU}(3)$ prediction is that the Primakoff excitation $\Sigma^{-} \rightarrow \Sigma^{*-}$ is forbidden, while excitation of $\Sigma^{+} \rightarrow \Sigma^{*+}$ is allowed. The U-spin values of interest are $\mathrm{U}=1 / 2$ for $\Sigma^{-}, \Sigma^{+}, \Sigma^{+}(1385)$ and $\mathrm{U}=3 / 2$ for $\Sigma^{-}(1385)$.

The E1 and M1 $\Sigma$ transitions are related to the intrinsic electric and magnetic $\Sigma$ polarizabilities. We will elucidate below this connection, which can be tested experimentally. Hadron electric $(\alpha)$ and magnetic $(\beta)$ intrinsic polarizabilities characterize the induced transient dipole moments. During $\gamma$-hadron Compton scattering, the lowest order scattering (Thomson) is determined by the charge and magnetic moment. The next order scattering (Rayleigh) is determined by the induced dipole moments. The Compton cross section data determine the Compton polarizabilities $\bar{\alpha}$ and $\bar{\beta}$, expressed here in Gaussian units of $10^{-43} \mathrm{~cm}^{3}$. The angular distribution formulae for low $\gamma$-ray energies in terms of $\bar{\alpha}$ and $\bar{\beta}$ for $\gamma \mathrm{p}$ scattering are given by Petrunkin and Lvov [4,5], and similar expressions apply for the $\Sigma$ hyperons. Perturbation theory for the $\Sigma^{+}, \Sigma^{-}$, and proton polarizabilities can be applied, leading to the expressions [4] of Petrunkin:

$$
\begin{aligned}
& \bar{\alpha}=\frac{2 \sum\left|\left\langle 0\left|\boldsymbol{d}_{\boldsymbol{z}}\right| n\right\rangle\right|^{2}}{E_{n}-E_{0}}+\frac{1}{3} \frac{e^{2}}{M}\left\langle r^{2}\right\rangle=\alpha+\Delta \alpha, \\
& \bar{\beta}=2 \sum \frac{\left|\left\langle 0\left|\boldsymbol{\mu}_{\boldsymbol{z}}\right| n\right\rangle\right|^{2}}{E_{n}-E_{0}}-k \frac{e^{2}}{M}\left\langle r^{2}\right\rangle=\beta+\Delta \beta .
\end{aligned}
$$

Here $\boldsymbol{d}=\sum e_{k} \boldsymbol{r}_{\boldsymbol{k}}$ and $\boldsymbol{\mu}$ are the electric and magnetic dipole operator respectively. We obtained [11] $\mathrm{k}=0.68,0.60,0.26$, and we use rms charge radii from lattice QCD calculations $[11,16]$ of $\mathrm{R}=0.86,0.96,0.76 \mathrm{fm}$, for the proton, $\Sigma^{+}$, and $\Sigma^{-}$respectively. The first and second terms in these equations give the intrinsic and center of charge oscillation contributions, respectively. The sums are over all E1 and M1 excitations. The intrinsic polarizabilities probe the internal structure of baryons and mesons.

Many theoretical and experimental polarizability studies have been made for the proton and neutron [17,18]. In the proton polarizability calculation of Weiner and Weise [18], the intrinsic part is mainly due to the charged pion or kaon cloud surrounding the proton core. Only two calculations were reported for the $\Sigma$. One is a simple non-relativistic quark bag calculation 
$[4,11]$ with no cloud. The second is via heavy baryon chiral perturbation theory [9]. Calculations are in progress by Lvov [5] for the Sigma Compton scattering with a dispersion relationship approach. Such an approach is needed to account for the effects of inelastic reactions such as $\gamma \Sigma \rightarrow \pi \Lambda$.

The odd parity $\Sigma^{*} 1 / 2^{-}$and $\Sigma^{*} 3 / 2^{-}$states near $520 \mathrm{MeV}$ excitation above the nucleon have the s orbit strange quark promoted to the p orbit; and also excitations of the nonstrange quarks. The intrinsic contribution of eqn. 1 can be evaluated using closure by saturating the sum over these states, giving $[2,11]$ :

$$
\alpha_{\Sigma^{+}} \sim \frac{2}{\Delta E}\left\langle\Sigma^{+}\left|\left(\sum q_{i} z_{i}\right)^{2}\right| \Sigma^{+}\right\rangle^{2} \sim \frac{2}{3} \frac{\left\langle r^{2}\right\rangle}{\Delta E} e^{2}\left(\frac{4}{9}+\frac{4}{9}+\frac{1}{9}\right) \approx 17.1 .
$$

The intrinsic magnetic polarizability can be evaluated by saturating the magnetic dipole excitations with the $\Sigma^{+}$to $\Sigma(1385)$ transition, giving $[4,11]$ :

$$
\beta_{\Sigma^{+}} \sim \frac{2}{M_{\Sigma^{*+}}-M_{\Sigma}}\left|\left\langle\Sigma^{+}\left|\boldsymbol{\mu}_{\boldsymbol{z}}\right| \Sigma^{*}\right\rangle\right|^{2} \sim \frac{2}{M_{\Sigma^{*+}}-M_{\Sigma}}\left(\frac{2 \sqrt{2}}{3} \mu_{\Sigma^{+}}\right)^{2} \sim 8.5,
$$

where $\mu_{\Sigma^{+}}$is the $\Sigma^{+}$magnetic moment. The $\Sigma^{+}$to $\Sigma^{*}$ magnetic dipole transition matrix element is written in terms of the $\Sigma^{+}$magnetic moment, following the non-relativistic quark model. For $\beta_{\Sigma^{+}}$, the magnetic dipole transition to the $\Sigma^{*} 3 / 2^{+}$resonance saturates the magnetic dipole excitations, in analogy to the nucleon to $\Delta$ transition; the proton and $\Sigma^{+}$have the same matrix element expression as members of the same U-spin doublet. For the proton intrinsic magnetic polarizability $\beta_{p}$, the analog of eqn. 4 gives [4] $\beta_{p} \approx 7.4$.

Consider matrix elements for magnetic moment operators $\boldsymbol{\mu}$ between states having the quark constituents of nucleons and $\Sigma$ 's. In the SU(3) symmetry limit, the contributions to the magnetic moment of the odd $d$ quark to $\mu_{p}$ and of the odd $s$ quark to $\mu_{\Sigma}$ are equal. Consider experimental tests of $\mathrm{SU}(3)$ breaking mechanisms based on the assumptions that the $u$ flavor contributions to $\mu_{p}$ and $\mu_{\Sigma^{+}}$are equal, and that $S U(3)$ breaking only occurs in $\boldsymbol{\mu}_{s}$. One can then show [11] that the strange contribution to the magnetic moment is suppressed by an order of magnitude:

$$
\frac{\mu_{\Sigma^{+}}+2 \mu_{\Sigma^{-}}}{\mu_{p}+2 \mu_{n}} \approx \frac{\left\langle\Sigma^{+}\left|\mu_{s}\right| \Sigma^{+}\right\rangle}{\left\langle p\left|\mu_{d}\right| p\right\rangle} \approx 0.11 \pm 0.04
$$

The main physical difficulty is in the small value of the $d$ quark contribution to the proton moment and the large contribution $2.45 \mathrm{n} . \mathrm{m}$. of the $u$ quarks. If the $u$ quark contribution to $\mu_{\Sigma^{+}}$is the same $2.45 \mathrm{n} . \mathrm{m}$. as in the proton, then the experimental value $\mu_{\Sigma^{+}}=2.42 \pm 0.02 \mathrm{n} . \mathrm{m}$. can be fit only by requiring a nearly vanishing $s$ quark contribution. That the strange quark contribution to $\mu_{\Sigma}$ is suppressed can also be seen by comparing the strange 
contributions to $\mu_{\Sigma}$ and $\mu_{\Lambda}$. The contribution to $\mu_{\Sigma}$ is only $6 \%$ of the strange contribution to $\mu_{\Lambda}[11]$.

Experimental data on related processes may give additional insight. One such process involves octet-decuplet transitions for nucleons and $\Sigma$ 's. It will be interesting to measure these transitions and compare their systematics with those of the magnetic moments. In a description of SU(3) symmetry breaking in the $\Sigma^{-}$to $\Sigma^{*-}$ transition, the ratio of M1 decay widths or of intrinsic magnetic polarizabilities for the $\Sigma^{-}$and proton can be estimated as $[10-12]$ :

$$
\frac{\Gamma\left(\Sigma \rightarrow \Sigma^{*-}\right)}{\Gamma\left(p \rightarrow \Delta^{+}\right)}=\frac{\left(M_{\Sigma^{*^{-}}}-M_{\Sigma^{-}}\right) \beta_{\Sigma^{-}}}{\left(M_{\Delta^{+}}-M_{p}\right) \beta_{p}} \approx \frac{1}{9}\left(1-\frac{\mu_{s}}{\mu_{d}}\right)^{2} \approx 0.011 .
$$

Here $\mu_{s}$ and $\mu_{d}$ are empirical magnetic moments of s and d quarks (estimated in a simple additive quark model [19] using proton, neutron, and Lambda magnetic moments, as $\left.\mu_{u}=1.852 \mu_{N}, \mu_{d}=-0.972 \mu_{N}, \mu_{s}=-0.613 \mu_{N}\right)$, the s and d values unequal due to $\mathrm{SU}(3)$ symmetry breaking. As shown in eqn. $5, \mu_{s}$ may be significantly lower than the value fixed by the Lambda magnetic moment; which would significantly increase the predicted decay width of eqn. 6 . Following the assumptions underlying eqns. 5-6, the $\Sigma^{-}$ M1 transition width directly determines the relative magnitudes of the $\mathrm{s}$ and d-quark magnetic moments. Explicit quark model calculations [20] give a larger value of 0.034 for the ratio of eqn. 6 , with $\Gamma\left(\Sigma^{*+}\right)=104 \mathrm{keV}[20]$ and $117 \mathrm{keV}$ [21]; and $\Gamma\left(\Sigma^{*-}\right)=2.5 \mathrm{keV}$ [20]. These calculations correspond to $\Gamma\left(\Delta^{+}\right)=74.3 \mathrm{keV}$ using eqn. 15 of Ref. 11 . The $\Delta$ radiative width of the model is smaller than the experimental value, but this should not affect the reliability of the ratio calculation of eqn. 6. One expects from eqns. (1$4,6)$ to observe very large and very small values of the intrinsic magnetic polarizability for the $\Sigma^{+}$and $\Sigma^{-}$, respectively; and similarly for the $\Sigma^{*}$ radiative decay width. The predictions [11] $\overline{\alpha_{\Sigma^{+}}}=20.8, \overline{\alpha_{\Sigma^{-}}}=5.7, \beta_{\Sigma^{+}}=$ $8.5, \beta_{\Sigma^{-}}=0.12, \overline{\beta_{\Sigma^{+}}}=1.7, \overline{\beta_{\Sigma^{-}}}=-1.7$ satisfy this expectation. Bernard et al. [9] predict quite different values.

The $\Sigma$ beam at FNAL is polarized, so that asymmetry measurements are possible; as in the recent study [22] of the $\Sigma \rightarrow p \gamma$ weak decay. Asymmetry data can also be of value for the $\Sigma$ polarizability determination. For radiative transitions, they are sensitive to the $\mathrm{M} 1$ or $\mathrm{E} 2$ nature of the exchanged photon, and therefore to the $\mathrm{L}=2$ admixtures. The ratio E2/M1 of these amplitudes is a subject of considerable current interest $[23,24]$ for the nucleon to $\Delta$ transition; so that measuring this ratio for the $\Sigma^{*}$ (and $\left.\Xi^{*}\right)$ will be extremely valuable. It was suggested $[2,11]$ that the ratios may be different for $\Sigma^{*}, \Xi^{*}$, and $\Delta$, given that the s-quark mass is significantly heavier than the $\mathrm{d}$ quark mass. Recent calculations $[25,26]$ give predictions for such transitions.

We consider now the event rate expectations and backgrounds for the $\Sigma$ polarizability and radiative transition experiments. We give the signal and 
background rates for a $\mathrm{C}^{12}$ target. For higher $\mathrm{Z}$ targets, the $\mathrm{Z}^{2}$ dependence of the Primakoff cross section compared to the $\mathrm{A}^{2 / 3}$ dependence of the strong cross section backgrounds, will improve the situation. We take a t-interval up to $6.0 \times 10^{-3} \mathrm{GeV}^{2}$, where $\mathrm{t}$ is the four momentum transfer to the target nucleus, as discussed in section 3 . We compare the $\Sigma$ expected rates to data and calculations for the $\pi \rightarrow \rho \gamma$ radiative transition measurement [27] at $156 \mathrm{GeV}$ on a $\mathrm{C}^{12}$ target. For the $\rho$ to $\pi$ transition, for this t-interval, the Primakoff cross section was $2.4 \mu \mathrm{b}$. and the strong background cross section was $0.75 \mu \mathrm{b}$; which gives a signal to background ratio of 3 . The hadronic cross section falls $[12,28]$ as $1 / \mathrm{E}$ (factor $=1 / 3.8$ ), while the Primakoff cross section rises as $\ln (\mathrm{E})$ (factor $=1.3$ ). In addition, the Primakoff cross section has a coefficient $\mathrm{K}$, given by:

$$
K=\frac{2 J_{a}^{*}+1}{2 J_{b}^{*}+1}\left(\frac{M_{a}^{*}}{M_{a *}^{2}-M_{a}^{2}}\right)^{3} \Gamma_{a^{*} \rightarrow a \gamma} .
$$

Considering mass and spin values, the coefficient $\mathrm{K}$ is 7 times larger for the $\Sigma^{*} \rightarrow \Sigma$ transition, compared to the $\rho \rightarrow \pi$ transition. We take [11] also the widths $\Gamma\left(\Sigma^{+}\right)=1000 \mathrm{KeV}$, and $\Gamma\left(\Sigma^{-}\right)=25 \mathrm{KeV}$. These are fixed by the widths given above, normalized to the experimental width of the $\Delta \rightarrow$ $\mathrm{N}$ radiative transition. The theoretical $\Delta$ width calculation is roughly 10 times lower than the experimental value. We assume here that the model calculation of width ratios is more accurate than the absolute values. We also assume that the strong backgrounds for the $\rho$ transition are of the same order as those of the $\Sigma$ transition. We obtain therefore the rough estimate of signal cross sections of 314 microbarns $\left(\Sigma^{+}\right)$, 7.8 microbarns $\left(\Sigma^{-}\right)$, with a background cross section of 0.2 microbarns. The count rates expected are very good, via comparison to the rates explicitly given later for pion polarizability, with a roughly 4.0 microbarn cross section. Furthermore, the width of the mass distribution for diffractive production of $\Sigma \rightarrow \Lambda \pi^{-}$is roughly $100 \mathrm{MeV}$, as shown in previous data, and calculations [29-31] for this process at BNL. Since the $\Sigma^{*}$ width is $36 \mathrm{MeV}$, and our mass resolution is $10 \mathrm{MeV}$, we will gain another factor of 2.5 from a mass cut.

We consider also the background from the small $\Xi^{-}$component in the beam. This decays $100 \%$ to $\Lambda \pi^{-}$, and is therefore a background for the $\Sigma^{*-}$ decay. The mass difference of $64 \mathrm{MeV}$ between $\Xi^{-}$and $\Sigma^{*-}$ is large compared to the expected mass resolution of $10 \mathrm{MeV}$. This background can therefore be distinguished in the off-line analysis. The on-line trigger may reduce this background, by requiring the vertex of the detected pion to be centered at the target position. Other background reactions have been considered, but will not be discussed here in detail. These include the high cross section process of $\Sigma^{-} \rightarrow \Sigma^{-} \pi^{0}$ diffractive dissociation; and the $\Sigma^{-} \rightarrow$ $n \pi^{-}$decay of beam particles. 


\section{Pion Polarizabilities and Radiative Transitions}

For the $\gamma-\pi$ interaction at low energy, chiral perturbation theory $(\chi \mathrm{PT})$ provides a rigorous way to make predictions; because it stems directly from QCD and relies only on the solid assumptions of spontaneously broken $\mathrm{SU}(3)_{L} \times \mathrm{SU}(3)_{R}$ chiral symmetry, Lorentz invariance and low momentum transfer. Unitarity is achieved by adding pion loop corrections to lowest order, and the resulting infinite divergences are absorbed into physical (renormalized) coupling constants $\mathrm{L}_{i}^{r}$ (tree-level coefficients in $\mathrm{L}^{(4)}$, see Refs. $([32,33])$. With a perturbative expansion of the effective Lagrangian limited to terms quartic in the momenta and masses $\left(\mathrm{O}\left(\mathrm{p}^{4}\right)\right)$, the method establishes relationships between different processes in terms of the $\mathrm{L}_{i}^{r}$. For example, the radiative pion beta decay and electric pion polarizability are expressed as: $[7,8,32,33]$ :

$$
h_{A} / h_{V}=32 \pi^{2}\left(L_{9}^{r}+L_{10}^{r}\right) ; \bar{\alpha}_{\pi}=\frac{4 \alpha_{f}}{m_{\pi} f_{\pi}^{2}}\left(L_{9}^{r}+L_{10}^{r}\right)
$$

where $\mathrm{f}_{\pi}=92.4 \mathrm{MeV}$ [34] is the pion decay constant, $\mathrm{h}_{A}$ and $\mathrm{h}_{V}$ are the axial vector and vector coupling constants in the decay, and $\alpha_{f}$ is the fine structure constant. The experimental ratio $\mathrm{h}_{A} / \mathrm{h}_{V}=0.45 \pm 0.06$, leads to $\bar{\alpha}_{\pi}=-\bar{\beta}_{\pi}=2.7 \pm 0.4$, where the error shown is due to the uncertainty in the $\mathrm{h}_{A} / \mathrm{h}_{V}$ measurement.

Other pion polarizability calculations [35-37] find values for $\bar{\alpha}_{\pi}$ ranging from $\sim 4-14$. Holstein [7] showed that meson exchange via a pole diagram involving the $\mathrm{a}_{1}(1260)$ resonance provides the main contribution $\left(\bar{\alpha}_{\pi}=2.6\right)$ to the polarizability. The E781 high energy pion experiments can obtain new high statistics data for radiative transitions leading from the pion to the $\mathrm{a}_{1}(1260)$, and to other meson resonances. For $\mathrm{a}_{1}(1260) \rightarrow \pi \gamma$, the experimental width [38] is $\Gamma=0.64 \pm 0.25 \mathrm{MeV}$. Xiong, Shuryak, and Brown (XSB) [39] estimate this radiative width to be $\Gamma=1.4 \mathrm{MeV}$, more than two times higher than the experimental value [38]. With this estimated width, they calculate the pion polarizability to be $\bar{\alpha}_{\pi}=1.8$. A remeasurement of the $\mathrm{a}_{1}(1260)$ width and of the pion polarizability will allow checking the consistency of their expected relationship.

For the pion polarizability, Antipov et al. [40,41] measured the $\gamma \pi$ scattering with $40 \mathrm{GeV}$ pions at Serpukhov via radiative pion scattering in the nuclear Coulomb field $\left(\pi^{-}+\mathrm{Z} \rightarrow \pi^{-}+\mathrm{Z}+\right.$ gamma). The final state gamma ray and pion were detected in coincidence. This reaction corresponded to $\gamma$ $+\pi^{-} \rightarrow \gamma+\pi^{-}$scattering for laboratory gamma-ray energies in the range $60-600 \mathrm{MeV}$ on a target $\pi^{-}$at rest. The data selection criteria at Serpukhov and E781 requires one photon and one charged particle in the final state, their total energy consistent with the beam energy, small t, and other position, angle, and energy conditions. Only the angular distribution in the backward hemisphere were measured at $40 \mathrm{GeV}$. The angular acceptance 
and detector threshold of the $\gamma$-ray calorimeter in E781 allows measuring more complete angular distributions than was achievable in the $40 \mathrm{GeV}$ experiment.

The pion electric polarizability $\overline{\alpha_{\pi}}$ was deduced $[40,41]$ in this low statistics experiment $(\sim 7000$ events $)$ to be $\bar{\alpha}_{\pi}=-\bar{\beta}_{\pi}=6.8 \pm 1.4_{\text {stat }} \pm 1.2_{\text {syst }}$, where it was assumed in the analysis that $\overline{\alpha_{\pi}}+\overline{\beta_{\pi}}=0$, as expected theoretically [7]. An important result of s-channel $(\gamma+\pi \rightarrow \gamma+\pi)$ dispersion sum rules [4] for charged pions is: $\bar{\alpha}_{\pi}+\bar{\beta}_{\pi}=0.39 \pm 0.04$. This result can be used for high statistics data as a constraint in the data analysis. Charged pion polarizabilities were also deduced [42] from $\gamma \gamma \rightarrow \pi^{+} \pi^{-}$data which is related to the Compton scattering by crossing symmetry. Pennington [43] claims that such determinations are very insensitive to the polarizability value. This is so, since two-photon cross section data at low $\pi \pi$ invariant mass agree well with calculations for a large range of choices of the (undetermined) position of the chiral zero. However, very different values of polarizability are associated with the different choices.

In the radiative pion scattering experiments, it was shown experimentally $[40,41]$ and theoretically [44] that the Coulomb cross section scales as $\mathrm{Z}^{2}$ and yields sharp peaks in t-distributions at very small four momentum transfers to the target nucleus $t \leq 6 \times 10^{-4}(\mathrm{GeV} / \mathrm{c})^{2}$. Background from other processes could easily be estimated and subtracted by extrapolating in $t$ from events in the region of flat t-distribution of $3-8 \times 10^{-3}(\mathrm{GeV} / \mathrm{c})^{2}$. The sources of these backgrounds are the coherent process of pion elastic scattering accompanied by gamma emission [44], contributions of pion (or rho) rescattering $[42,45]$, and higher cross section inelastic channels $[27,46]$. Available calculations and data show that these backgrounds are manageable, and that the signal to background improves $[12,28,44]$ with increasing incident energy.

One must also evaluate electromagnetic corrections to radiative pion scattering, where the requirement is to measure only single photon bremsstrahlung emission. Here the detailed properties of the gamma detector are important, such as the photon detector threshold, t-resolution, and the two-photon angular resolution. Such calculations were carried out $[47,48]$ for the conditions of the planned $600 \mathrm{GeV}$ FNAL experiment. The corrections are at the level of $4 \%$, and can be easily made.

The polarizability Compton scattering and chiral anomaly processes in E781 represents a difficult triggering challenge. They have one negative charged track in the final state, and one or two $\gamma$-rays. This trigger rate must match that of the charm component of the experiment. The trigger problems are deadtime problems generated in the initial trigger levels when information from the $\gamma$-ray detector is not available yet for trigger rate suppresssion. The first level of hardware trigger $T_{0}$ is meant to identify a beam particle and an interaction in the target. However, a $\mathrm{T}_{0}$ trigger with multiplicity 1 will generate a trigger for every beam particle, in contrast to other 
trigger types which will fire at the beam interaction rate. To differentiate between a single negatively-charged Primakoff interaction product coming from the target and a non-interacting beam track, it is planned to have three hodoscopes to measure a vertical deflection angle of the outgoing track after the Primakoff target. We need to form a coincidence between the projective elements of the first two hodoscopes to form the track road assuming a beam particle, and then veto in a window around this road in the third hodoscope. The required electronics must give the decision in about $140 \mathrm{nsec}$, in order to enter the next trigger level on time. A simpler beam prescale trigger will also be used; which will help control systematic uncertainties.

To specifically illustrate some of the kinematics germane to a FNAL experiment, the reaction:

$$
\pi+Z \rightarrow \pi^{\prime}+Z^{\prime}+\gamma^{\prime}
$$

is considered for a $600 \mathrm{GeV}$ incident pion, where $\mathrm{Z}$ is the nuclear charge. The 4-momentum of each particle is $P_{\pi}, P_{Z}, P_{\pi^{\prime}}, P_{Z^{\prime}}, k^{\prime}$, respectively. In the one photon exchange domain, eqn. 9 is equivalent to:

$$
\gamma+\pi \rightarrow \gamma^{\prime}+\pi^{\prime}
$$

and the 4-momentum of the incident virtual photon is $\mathrm{k}=P_{Z^{-}} P_{Z^{\prime}}$. The cross section for the reaction of eqn. 9 is described by the the well tested Primakoff formalism $[27,49]$ that relates processes involving real photon interactions to production cross sections involving the exchange of virtual photons. We have:

$$
\frac{d \sigma}{d t d s d \Omega}=\frac{Z^{2} \alpha_{f}}{\pi} \frac{|F(t)|^{2}}{s-m_{\pi}^{2}} \frac{t-t_{0}}{t^{2}} \frac{d \sigma_{\gamma \pi}}{d \Omega},
$$

where $\mathrm{d} \sigma_{\gamma \pi} / \mathrm{d} \Omega$ is the unpolarized differential cross section for eqn. 10 (for real photons), $t$ is the square of the four-momentum transfer to the nucleus, $\mathrm{F}(\mathrm{t})$ is the nuclear form factor (essentially unity at small t-values), $\sqrt{s}$ is the mass of the $\gamma \pi$ final state, and $t_{0}$ is the minimum value of $t$ to produce a mass $\sqrt{s}$. The analysis to determine polarizabilities by fitting the data use the known $[40-42,50]$ formula for $d \sigma_{\gamma \pi} / d \Omega$. This cross section depends on the polarizabilities and on $\mathrm{s}$ and on $\mathrm{t}_{1}$, the square of the 4-momentum transfer between initial and final state $\gamma$ 's. We have:

$$
t=k^{2} \equiv-M(V)^{2}
$$

where $\mathrm{k}$ is the 4-momentum transferred to the nucleus, and $\mathrm{M}(\mathrm{V})$ is the virtual photon mass. Since $t=2 M_{Z}\left(E_{Z^{\prime}, l a b}-M_{Z}\right)>0$, the virtual photon mass is imaginary. To approximate real pion Compton scattering, the virtual photon must be almost real; $\mathrm{M}(\mathrm{V})<0.077 \mathrm{GeV}$ corresponding to $t<6.0 \times$ $10^{-3}(\mathrm{GeV} / \mathrm{c})^{2}$ can be required in E781. In addition, 


$$
s=\left(P_{\pi^{\prime}}+k^{\prime}\right)^{2} \equiv M(\gamma \pi)^{2}
$$

where $M(\gamma \pi)$ is the $\gamma \pi$ invariant mass. The minimum value for $\mathrm{t}[51]$ is given by:

$$
t_{0} \sim\left(s-m_{\pi}^{2}\right)^{2} / 4\left|\boldsymbol{P}_{\boldsymbol{\pi}}\right|^{2},
$$

corresponding to $t_{0} \sim 5.4 \times 10^{-8}(\mathrm{GeV} / \mathrm{c})^{2}$ for $\sqrt{s}=1.75 m_{\pi}$ at $600 \mathrm{GeV}$ incident energy. The maximum of the differential cross section for reaction (11) occurs at $t=2 t_{0}$, and the integral to $100 t_{0}$ gives essentially the entire cross section.

With lead glass detectors [40,41], a t-resolution $\sigma_{t} \sim 6.0 \times 10^{-4}(\mathrm{GeV} / \mathrm{c})^{2}$ was achieved at $40 \mathrm{GeV}$. The t-resolution sets the experimental maximum in $\mathrm{t}$ for accepted events. The SELEX t-resolution will be 10 times worse. The strong backgrounds are associated with particle exchange, and such cross sections are known $[12,27,28,44]$ to fall rapidly with increasing energy. From this point of view, the t-resolution is therefore not a critical parameter for $600 \mathrm{GeV}$ experiments. The integrated Compton cross section up to $t=$ $6.0 \times 10^{-3}(\mathrm{GeV} / \mathrm{c})^{2}$ grows $[44,51]$ as $\ln \left(\boldsymbol{P}_{\boldsymbol{\pi}}\right)$, where $\boldsymbol{P}_{\boldsymbol{\pi}}$ is the laboratory incident pion 3-momentum. With strong backgrounds that decrease roughly as $1 / \mathrm{E}$, the percent strong background would decrease for a $\mathrm{C}^{12}$ target, from the estimated [44] $2.5 \%$ at $40 \mathrm{GeV}$ to roughly $0.6 \%$ at $600 \mathrm{GeV}$ at FNAL.

The energy of the incident virtual photon in the pion rest frame is:

$$
E(V)=\left(s-m_{\pi}^{2}+t\right) / 2 m_{\pi} \sim\left(s-m_{\pi}^{2}\right) / 2 m_{\pi}
$$

at small t; so that the energy of the photon is determined by s. The elemental cross section at low $\mathrm{E}(\mathrm{V})$ is a function of $\mathrm{E}(\mathrm{V}), \cos (\theta), \bar{\alpha}, \bar{\beta}$; where $\theta$ is the Compton scattering angle in the pion rest frame. In this frame, due to Lorentz contraction, the nucleus $\mathrm{Z}$ represents a transverse virtual photon pulse sweeping past the pion. One should require that $E(V)$ be sufficiently low in energy, such that $\rho$ meson production does not occur on-shell for an incident $\gamma$-ray on a target pion at rest. The $\gamma$-ray energy required to produce a $\rho$ meson, not counting the $\rho$ width, is given by $\omega=E(V)=$ $\left(M_{\rho}^{2}-m_{\pi}^{2}\right) /\left(2 m_{\pi}\right)=2.0 \mathrm{GeV}$. Considering the $\rho$ width, one could limit the incident energy to be lower than $1 \mathrm{GeV}$, corresponding to $\mathrm{s}<15.3 m_{\pi}^{2}$. We will analyze the lower energy data for polarizability purposes, and at higher energies to understand the $\rho$ and $\mathrm{a}_{1}$ meson contributions.

Consider the case of $600 \mathrm{GeV}$ incident laboratory pions. The laboratory outgoing $\gamma$-rays emitted up to $5 \mathrm{mrad}$, and the corresponding outgoing pions emitted up to $0.3 \mathrm{mrad}$. The angular resolution for the pion is roughly 0.04 mrad due to multiple scattering in the Primakoff target and in the in-beam tracking detectors. The gamma ray energies range from $0-400 \mathrm{GeV}$, and the corresponding outgoing pion energies range from $200-600 \mathrm{GeV}$. The corresponding Compton scattering angular range is 0 - 180 degrees in the $\pi$ rest frame. In practice, the most forward Compton scattering angles are less accessible, as they correspond to the larger $\gamma$-ray angles in the laboratory 
frame which can miss the $\gamma$-ray detector, and where the $\gamma$-ray energies are also possibly below the detector threshold. But these forward angles are anyhow not sensitive to the polarizabilities, as discussed below.

We consider the uncertainties achievable for the polarizabilities in the FNAL E781 experiment, based on Monte Carlo simulations. An important consideration is the information content $[3,51]$ of the data versus $\mathrm{x}$ and $\mathrm{s}$, where $\mathrm{x}=\cos (\theta)$. Consider the fraction of the $\gamma \pi$ Compton cross section arising from the $\bar{\alpha}$ terms in the $\gamma \pi$ center of mass cross section expression, for $\bar{\alpha}=6.8$. High s values and back angles (large $t_{1}$ ) have maximal information content for the polarizabilities. For example, the fraction of the cross section at back angles due to the polarizability term is only $5 \%$ at $\mathrm{E}(\mathrm{V})=140 \mathrm{MeV}$, and roughly $30 \%$ at $\mathrm{E}(\mathrm{V})=600 \mathrm{MeV}$.

We consider initially a beam energy of $600 \mathrm{GeV}$, a $\mathrm{C}^{12}$ target, and an s-range of 2. - $10 . \mathrm{m}_{\pi}^{2}$, corresponding to a Primakoff cross section of $4.0 \mu \mathrm{b}$, and $\mathrm{E}(\mathrm{V})=70-628 \mathrm{MeV}$. We take the $\pi$-Carbon total cross section at 600 $\mathrm{GeV}$ to be $192 \mathrm{mb}$, eight times the total inelastic $\pi$-nucleon cross section at $600 \mathrm{GeV}$. We assume the simultaneous use of a 5\% Carbon interaction target and also a $0.3 \% \mathrm{~Pb}$ interaction target. We calculate below the event rate from the Carbon target, and will in addition have a factor of roughly 2 times higher rate from the $\mathrm{Pb}$ target. The probability $\mathrm{P}$ per inelastic interaction for a Primakoff interaction is then $P=4 . \times 10^{-6} / 192 . \times 10^{-3}=2.1 \times 10^{-5}$. The number of interactions planned [1] for E781 is roughly $3 . \times 10^{10}$, corresponding to roughly $6.3 \times 10^{5}$ events for $4.0 \mu \mathrm{b}$. Including the $\mathrm{Pb}$ target events, the statistics are roughly 3 times higher. The trigger requirement for a vertical deflection angle of the pion of more than $100 \mu \mathrm{rad}$ cuts the statistics roughly in half. The losses are mainly events at small $\mathrm{s}$ and $\mathrm{t}_{1}$, which minimally affects polarizability uncertainties. Other experimental efficiencies and acceptances will lower the statistics.

We cite here some Monte Carlo results for $600 \mathrm{GeV}$ Primakoff experiments on Carbon, assuming the dispersion sum rule result $\bar{\alpha}+\bar{\beta}=0.4$ and also $\bar{\alpha}=6.8$. At $600 \mathrm{GeV}$, including the $\mathrm{Pb}$ target, for 580,000 events in an $\mathrm{s}$ interval $(2.0-10.0) \mathrm{m}_{\pi}^{2}$, we find by fitting simulated data that $\bar{\alpha}=7.1 \pm$ $0.4, \bar{\alpha}+\bar{\beta}=0.3 \pm 0.1$. The overall statistical error for polarizabilities will be improved from the expected higher statistics; and by additional data in the sensitive s-interval 10.-15.3 $\mathrm{m}_{\pi}^{2}$. Data in different s-intervals can be analyzed to give independent values for the polarizabilities, which will help control the systematic uncertainties. These Monte Carlo simulations show that the objective of obtaining pion polarizabilities with significantly smaller statistical and systematic uncertainties is realistic. 


\section{Conclusions}

The beams at FNAL and CERN invite hadron Compton scattering and radiative transition studies for different particle types, such as $\pi^{+,-}, K^{+,-}$, p, $\bar{p}, \Sigma, \Xi, \Lambda$ hyperons, and others. The E781 experiment was described. Because these transitions involve the well understood electromagnetic process, they provide precision tests of the quark wave functions characterizing the configurations of the low lying excited states of hadrons. The $600 \mathrm{GeV}$ beam energy at FNAL is important to get a good yield for low t events in the radiative scattering, and also to reduce backgrounds from the decay of unstable hadrons by significantly boosting their lifetime. We will measure the $\gamma \pi$ and $\gamma \Sigma$ Compton scattering cross sections, thereby enabling determinations of the pion and Sigma polarizabilities. E781 will also measure the formation and decay of the $\Sigma^{-}(1385), \Sigma^{+}(1385)$, and $a_{1}(1260)$ excited states. These various $\Sigma$ and pion experiments will allow serious tests of chiral symmetry and chiral perturbation theory; and of different available polarizability and radiative decay calculations in QCD. The $\Sigma$ experiments will shed new light on puzzles associated with the size of the s-quark magnetic moment.

\section{Acknowledgements}

This work was supported by the U.S.-Israel Binational Science Foundation, Jerusalem, Israel. Thanks are due to S. Bellucci, P. Cooper, T. Ferbel, L. Frankfort, S. Gerzon, G. Giordano, A. Kulyavtsev, J. Lach, H. J. Lipkin, A. Lvov, J. Russ, and N. Terentyev for valuable discussions.

\section{References}

1. J. Russ, spokesman, FNAL E781 Collaboration: Carnegie-Mellon U., Fermilab, U. Iowa, U. Rochester, U. Washington, Petersburg Nuclear Physics Institute, ITEP (Moscow), IHEP (Protvino), Moscow State U., U. Sao Paulo, Centro Brasileiro de Pesquisas Fisicas, Universidade Federale de Paraiba, IHEP (Beijing), U. Bristol, Tel Aviv U., Max Planck Institut fur Kernphysik-Heidelberg, Universidad Autonoma de San Luis Potosi;

J. Russ: Proceedings of the CHARM2000 Workshop, Fermilab, June 1994, Eds. D. M. Kaplan and S. Kwan, Fermilab-Conf-94/190, P. 111, (1994)

2. M. A. Moinester: Proceedings of the Conference on the Intersections Between Particle and Nuclear Physics, Tucson, Arizona, 1991, AIP Conference Proceedings 243, P. 553, 1992, Ed. W. Van Oers.

3. M.A. Moinester: Workshop on Hadron Structure from Photo-Reactions at Intermediate Energies, Brookhaven National Laboratory, May 1992, Eds. A. Nathan, A. Sandorfi, BNL Report 47972, Tel Aviv U. preprint TAUP $1972 / 92$. 
4. V. A. Petrunkin: Sov. J. Part. Nucl. 12278 (1981)

5. A. I. Lvov: Sov. J. Nucl. Phys. 42583 (1985); Int. J. Mod. Phys. A 85267 (1993); Phys. Lett. B 30429 (1993) ; private communication.

6. J. L. Friar: Workshop on Electron-Nucleus Scattering (1988, EIPC), Eds.

A. Fabrocini et al., World Scientific Publishing Co. (1989)

7. B. R. Holstein: Comments Nucl. Part. Phys. 19239 (1990)

8. J. F. Donoghue, B. R. Holstein: Phys. Rev. D 402378 (1989)

9. V. Bernard et al: Phys. Rev. D 462756 (1992); Phys. Lett. B 319269 (1993)

10. H. J. Lipkin: Phys. Rev. D 7846 (1973)

11. H. J. Lipkin, M. A. Moinester: Phys. Lett. B 287179 (1992)

12. A. V. Vanyashin et al: Sov. J. Nucl. Phys. 3490 (1981)

13. T. Goldman et al: Physics with LAMPF II, LA-9798-P, P. 319 (1984)

14. M. V. Hynes: Physics with LAMPF II, LA-9798-P, P. 333 (1984)

15. F. Dydak et al: Nucl. Phys. B 1181 (1977)

16. D. B. Leinweber, R. M. Woloshyn, T. Draper: Phys. Rev. D 431659 (1991)

D. B. Leinweber, T. D. Cohen: Phys. Rev. D 472147 (1993)

17. B. R. Holstein, A. M. Nathan: Phys. Rev. D 496101 (1994)

18. R. Weiner, W. Weise: Phys. Lett. B 15985 (1985)

19. Particle Data Group, G.P.Yost et al: Phys. Lett. B 2041 (1988)

20. J. W. Darewych et al: Phys. Rev. D 281125 (1983)

21. E. Kaxiras, E. J. Moniz, M. Soyeur: Phys. Rev. D 32 695, (1985)

22. M. Foucher et al: Phys. Rev. Lett. 683004 (1992)

23. R. M. Davidson, N. C. Mukhopadhyay, R.S. Wittman: Phys. Rev. D 4371 (1991)

24. A. Bernstein, S. Nozawa, M. A. Moinester: Phys. Rev. C 471274 (1993)

25. D. B. Leinweber, T. Draper, R. M. Woloshyn: Phys. Rev. D 482230 (1993)

26. M. N. Butler, M. J. Savage, R. P. Springer: Phys. Lett. B 304353 (1993);

Phys. Lett. 314 122(E)(1993); Nucl. Phys. B 39969 (1993)

27. T. Jensen et al: Phys. Rev. D 2726 (1983)

28. G. Berlad et al: Ann. Phys. 75461 (1973)

29. R. T. Deck: Phys. Rev. Lett. 13169 (1964)

30. L. Stodolsky: Phys. Rev. Lett. 18973 (1967)

31. V. Hungerbuhler et al: Phys. Rev. D 102051 (1974)

32. J.Gasser, H.Leutwyler: Nucl. Phys. B 250465 (1985)

33. J.Gasser, H.Leutwyler: Ann.Phys. (N.Y.) 158142 (1984)

34. B.R.Holstein: Phys.Lett.B 24483 (1990)

35. V. Bernard, B. Hiller, W. Weise: Phys. Lett. B 15985 (1988)

36. V. Bernard, D. Vautherin: Phys. Rev. D 401615 (1989)

37. M. A. Ivanov, T. Mizutani: Phys. Rev. D 451580 (1992)

38. M. Zielinski et al: Phys. Rev. Lett. 521195 (1984)

39. L. Xiong, E. Shuryak, G. Brown: Phys. Rev. D 463798 (1992)

40. Y. M. Antipov et al: Phys. Lett. B 121445 (1983)

41. Y. M. Antipov et al: Z. Phys. C-Particles and Fields 26495 (1985)

42. D. Babusci, S. Bellucci, G. Giordano, G. Matone, A. M. Sandorfi, M. A. Moinester: Phys. Lett. B 277158 (1992) 
14 Murray A. Moinester: Pion and Sigma Polarizabilities and Radiative Transitions

43. J. Portoles, M. R. Pennington: U. Durham preprint DTP-94/52, 1994, submitted to Second DA $\Phi$ NE Physics Handbook, Eds. G. Pancheri and N. Paver.

44. A. S. Galperin et al: Sov. J. Nucl. Phys. 32545 (1980)

45. G. V. Mitselmakher, V. N. Pervushkin: Sov. J. Nucl. Phys. 37 (1983)

46. M. Zielinski et al: Z. Phys. C 16197 (1983)

47. A. A. Akhundov, D. Yu. Bardin, G. V. Mitselmakher, A. G. Olshevsky: Sov. J. Nucl. Phys. 42426 (1984)

48. A. A. Akhundov, S. Gerzon, S. Kananov, M. A. Moinester: I.C.T.P. (Trieste) Preprint IC/94/203; Tel Aviv U. Preprint TAUP-2183-94, 1994

49. M. Zielinski et al: Phys. Rev. D 292633 (1984)

50. L. V. Fil'kov, I. Guiasu, E. E. Radescu: Phys. Rev. 263146 (1982)

51. N. I. Starkov, L. V. Fil'kov, V. A. Tsarev: Sov. J. Nucl. Phys. 36707 (1982)

This book was processed by the author using the $\mathrm{T}_{\mathrm{E}} \mathrm{X}$ macro package from Springer-Verlag. 